\title{
Machine learning in the problem of recognition of pitting corrosion on aluminum surfaces
}

\author{
M Enikeev ${ }^{1}$, L Enikeeva' ${ }^{1}$, M Maleeva $^{2}$ and I Gubaydullin ${ }^{1,3}$ \\ ${ }^{1}$ Ufa State Petroleum Technological University, Kosmonavtov St. 1, Ufa, Russia, 450062 \\ ${ }^{2}$ A.N. Frumkin Institute of Physical Chemistry and Electrochemistry Russian Academy of \\ Sciences, Leninsky prospect 31, Moscow, Russia, 199071 \\ ${ }^{3}$ Institute of Petrochemistry and Catalysis, Russian Academy of Sciences, Prospect Oktyabrya \\ 141, Ufa, Russia, 450075
}

\begin{abstract}
The work is devoted to the problem of identification and quantitative estimation of pitting corrosion. The purpose of the work is to design an algorithm and create a set of programs for evaluating and predicting corrosion processes development on the aluminum surface. Object of a research is process of corrosion of aluminum with hydrogen depolarization. Results of work can be used for a research of corrosion processes and their mechanisms on the basis of the visual analysis.
\end{abstract}

\section{Introduction}

One of the reasons of decrease in durability and loss of metal designs operability is corrosion destruction of metal. Most of all iron, its alloys and also aluminum suffer from corrosion. Metal designs are subject to generally electrochemical corrosion which arises at contact of metals with electrolytes. Diagnostics and forecasting of corrosion allow estimating service conditions of devices, machines and the equipment. Also they allow defining efficiency of methods and means of protection, to make the decision on their improvement.

Among the non-destructive testing methods for corrosion monitoring [1-4] are scanning reflectometry, confocal microscopy, optical microscopy, ultrasonic inspection, laser scanning microscopy, acoustic emission, vibration analysis, etc. Due to the ease of monitoring, visual inspection is still employed Due to the ease of monitoring, visual inspection is still employed with satisfactory results because it informs about the type and extent of the corrosion. Due to the ease of monitoring, visual inspection is still employed because it informs about the type and level of the corrosion. However, visual inspection has subjective criteria.

The most part of the works connected with detection of corrosion effects by methods of computer vision analyzes cracks and pittings. In most of works, the problem of automation detection and defects measurement was solve successfully. Studying of pitting corrosion of stainless steel in FeCl3 solution became one of the initial experience with computer vision for the metals corrosion research [5]. The ratio of the damaged area to the total image area was determined by photos of corrosion process. Corrosion studying as three-dimensional object, that is determination of depth and a damaged form by computer vision methods merits attention. [6]. In [7] other method of metal corrosion identification based on a minimum distance between the recognition objects is presented. Paper [8] considers fractal geometry methods for the analysis of corrosion process. Researchers don't take into account that the reliable application of image processing needs noise reduction. Abdel-Qader [9] has compared various 
edge detection algorithms and has revealed that the Haar's method is the best practice for crack detection. However on noisy data this method is likely to be inefficient as well as the methods based on morphological operations [10]. Prasanna [11] applied histogram analysis and support vector machine (SVM) in classification for the crack detection. The detection rate of the classifier has been found to be approximately $76 \%$. In paper [12] applied the principal component analysis (PCA) for search cracks on the bridge surface. In this case the accuracy of the analysis depends on camera position. Lattanzi and Miller [13] developed the clustering method based on k-means clustering algorithm and the Canny edge detector. In [14] developed algorithm that produces automatically and accurately the quantitative and objective information on the corrosion state of the electroless nickel immersion gold surface using factor analysis (metal turn over and $\mathrm{pH}$ of the plating bath). Belim and Kutlunin [15] developed the algorithm of boundary extraction based on image clustering. The mathematical model will allow to describe quantitatively processes on interphase boundaries in a wide number of test environments and metals. The model is based on image processing of corroding surface. The computer vision system can serve as the system realizing such model. In [16] developed design of the device for measurement of the depth of the surface defect of the controlled surface, for example. Vasin [17] describe a machine vision system for the inspection of the railway track condition.

The study of corrosion processes using computer vision methods involves four interconnected processes that create the prerequisites for the creation of data-processing system:

1. Accumulation of experimental data (corroded surface images), which require ordering, structuring and classification for correct use when solving the problem of classifier training.

2. Development of information processing methods depending on the type of problem being solved. In this case, it is the use of image processing methods, mathematical statistics and fractal analysis to evaluate and predict corrosion processes for various types of metals (steel, iron and aluminum).

3. Updating the technical means of processing information, increasing the calculations speed (using graphics processors).

4. Implementation of automatic systems of scientific research in the form of software packages and training of the end user (researcher).

In this paper, we study the results obtained with the help of the created information-computational analytical system (ICAS) for estimating and predicting corrosion ("CorOcenka"). The main results based on the use of ICAS are listed.

\section{Pitting corrosion identification}

The image of an aluminum surface under corrosion potential in a $0.1 \mathrm{M} \mathrm{NaCl}$ solution (pH 11) is shown in figure 1. Process is followed by intensive release of hydrogen. Bubbles of the evolving hydrogen are presented on the image. The task of searching objects in the image was reduced to detection of hydrogen bubbles being evolved. The intensity of this evolution had to be determined from a series of pictures. On the volume of the evolved hydrogen the rate of cathodic reaction is estimated. This rate is equal to the rate of anode reaction and dissolution or corrosion rate.

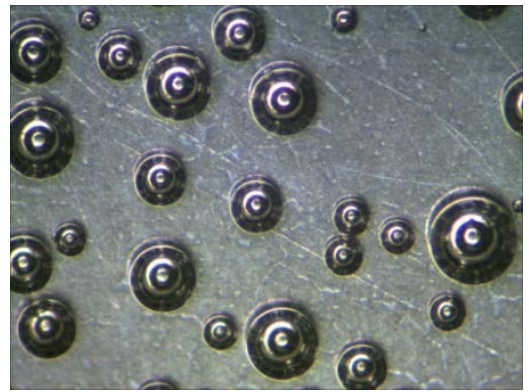

Figure 1. The snapshot of the aluminum surface at a corrosion potential in $0.1 \mathrm{M} \mathrm{NaCl}(\mathrm{pH} 11)$.

In the study of the corrosion reactions such methods of information processing as image processing, fractal analysis, contour analysis and template matching are used. 
Mathematical processing of experimental data consists in work with the image $f$ which is matrix $\mathrm{f}(\mathrm{M}, \mathrm{N})$ :

$$
f(x, y)=\left[\begin{array}{rccl}
f(0,0) & f(0,1) & \mathrm{K} & f(0, N-1) \\
f(1,0) & f(1,1) & \mathrm{K} & f(1, N-1) \\
\mathrm{M} \mathrm{M} & \mathrm{M} & \\
f(M-1,0) & f(M-1,1) & \mathrm{K} & f(M-1, N-1)
\end{array}\right]
$$

This matrix is mathematical model of the image. Each element of the matrix is image pixel.

The following steps can be distinguished in the recognition of surface defects:

1. Image pre-processing (anti-aliasing, noise filtering, increasing contrast);

2. Actual processing (image binarization and isolation of object contours);

3. Image post-processing (filtration of contours on perimeter and area, on form coefficient and fractality, etc).

These operations are specific to each problem of computer visioin. Development and use of the models suitable for the effective solution of a object detection and recognition problems, substantially remains on the verge of science and art. The solution of this problem requires a special know-how or, in other words, knowledge of subject domain reflecting long-term experience of a research on the solution of specific tasks. In more detail this task is considered in work [18].

For search of a bubble on the image we will use the Histogram of Oriented Gradients method (HOG, [19]) The method consists in assessment of normalized gradients histograms of the image brightness. The image is divided into small rectangular regions, in each of which brightness gradient is calculated. For all received gradients in the area the histogram is calculated. The histogram is the descriptor of the image region. For increase in accuracy local histograms use normalization by contrast. In order to take into account changes in illumination and contrast, the strength of the gradient must be locally normalized, which requires grouping the cells into larger, spatially related blocks. Because of its locality, the HOG method is stable to rotations.
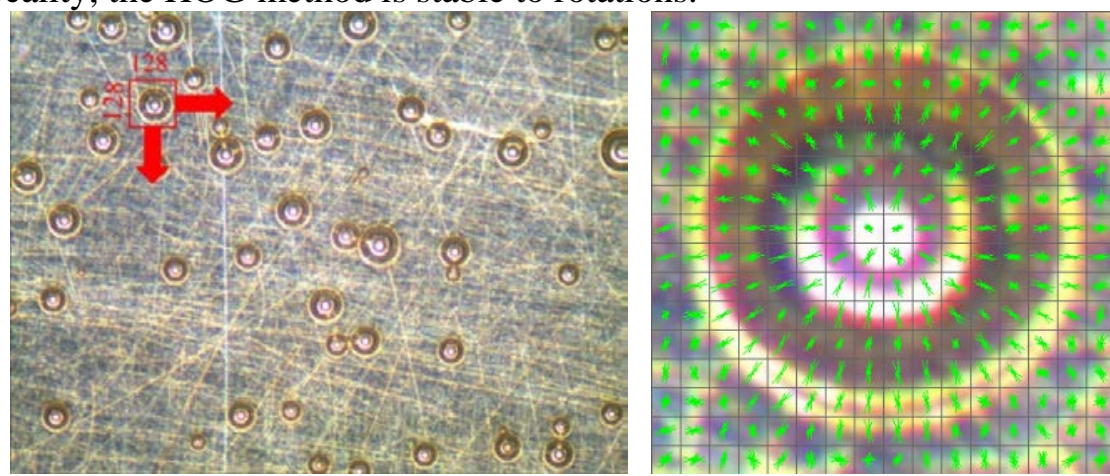

Figure 2. Visualization of the "sliding window" on the example of the image from the data set of the aluminum corrosion with hydrogen depolarization and the construction of oriented gradients histograms for a bubble on the aluminum surface.

We will search for a "bubble" in the image using a "sliding window" with a size of $128 \times 128$ pixels. We divide it into blocks of $8 \times 8$. Then one window with a size of $128 \times 128$ pixels will contain 256 blocks. In each block, we calculate the histogram of oriented gradients with 8 cells. Thus, we get 8 $\times 256=2048$ characteristics. In Fig. 2 the visualization of this method is presented, the bubble image has a size of $128 \times 128$ pixels and is divided into 256 blocks. For illustrative purposes in this case a scaled model is presented. Such a number of characteristics cannot be taken into account without the use of machine learning. An effective method for solving this problem is the support vector machine [20].

Machine learning is the extraction of knowledge from data. This is a scientific field combining the methods of statistics, artificial intelligence and computer science and also known as forecasting analytics or statistical training. The solution of the problem of binary classification using the support 
vector method consists in finding a linear function that correctly divides the data set into two classes. Consider the classification problem, where the number of classes is two.

The problem can be formulated as a search for a function $\mathrm{y}(\mathrm{x})$ that takes values greater than zero for vectors of the same class (for bubble images) and less than zero for vectors of another class (background). As the initial data for solving the problem, that is, searching for the classifying function $y(x)$, a training set of space vectors is given for which they are known to belong to one of the classes. The collection of classifying functions can be described in terms of the function $\mathrm{y}(\mathrm{x})$. The hyperplane is defined by the vector a and the value b, that is, $y(x)=a x+b$. As a result of the SVM-model construction, a function that takes values less than zero for vectors of one class and greater than zero for vectors of another class is found. For each new object, a negative or positive value determines whether the object belongs to one of the classes.

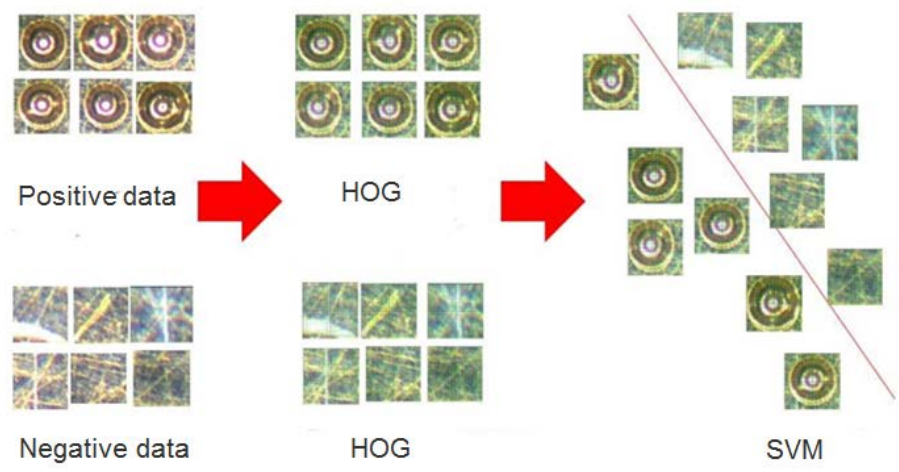

Figure 3. Training of SVM linear classifier.

The sequence of actions for training of the linear SVM qualifier consists of the following steps (Fig. 3):

- We will analyze the image by means of a window of $128 * 128$ pixels in size

- We will break it into blocks of $8 * 8$ in size. Then one window of $128 * 128$ pixels in size will contain 256 blocks.

- We will count the histogram of oriented gradients with 8 cells in each block.

- We receive $8 * 256=2048$ properties wn total.

After the objects in the image are found, in this case a bubble, we calculate its area. Since the contour of the bubble is a circle, then, using the method of least squares for the circle, we can calculate the necessary values for the radius and center of the bubble.

\section{Experiment Analysis}

The software package that implements ICAS "CorOcenka” was written in C ++ language in the Qt SDK environment using the OpenCV library, and SQLite was used as a database.

To solve the problem of corrosion analysis on the surface of aluminum, a sample was created, consisting of one hundred positive and negative examples.

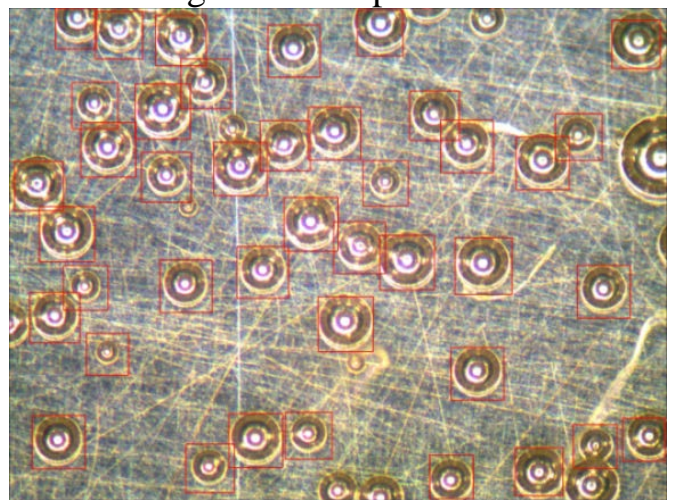

Figure 4. The result of the linear classifier work on the data of the initial experiment. 


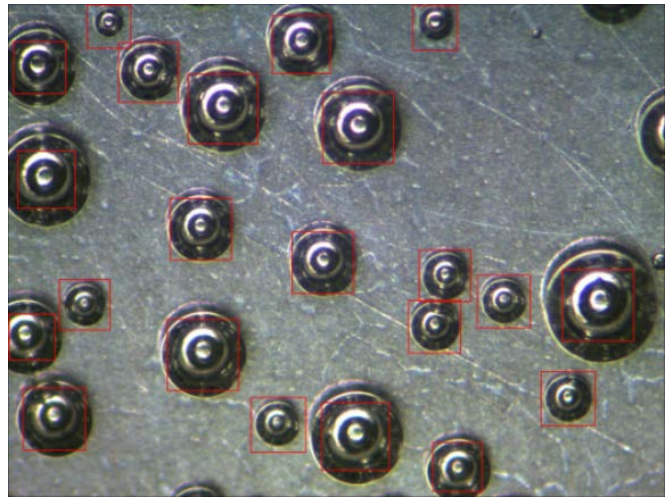

Figure 5. The result of the linear classifier work on the data of an alternative experiment.

On the sample, the SVM linear classifier was trained. Fig. 4 demonstrates that the classifier unsatisfactorily processes bubbles that partially fall into the frame or have small dimensions. This effect can be neglected to obtain an overall picture of the change in the area of the observed bubbles.However, to obtain an overall picture of the change in the area of the observed bubbles, this effect can be neglected.

To verify the performance of the classifier, its work was tested on a set of data from another experiment occurring under different conditions. The result of processing the experimental data is shown in Fig. 5. Despite the fact that the classifier was trained on the data of the first experiment, it showed good performance on an alternative data set.

The use of the parallel implementation of the SVM training had an excellent effect on the calculation time. In general, for the task of identifying bubbles on the image, the acceleration was more than 15 times.

The change in the total area of the bubbles as a function of time was obtained (Fig. 6).

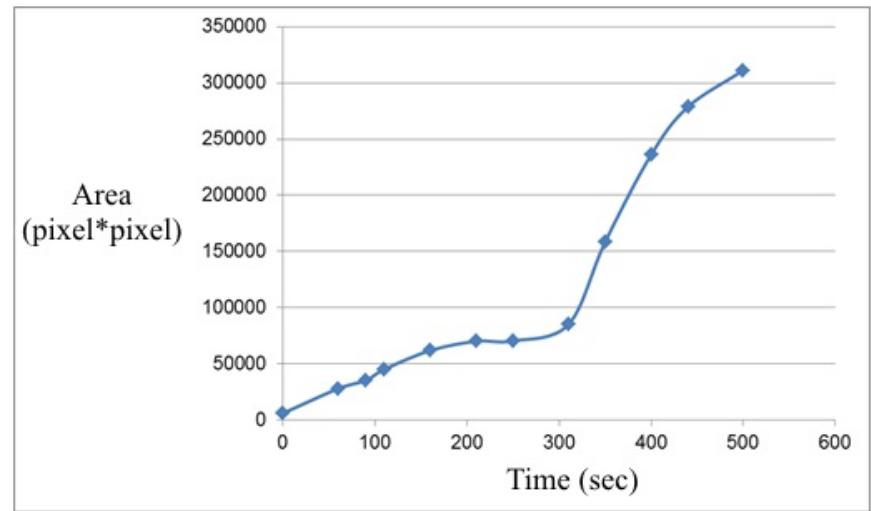

Figure 6. Change in bubble size over time.

Also the possibility of bubbles search by means of contour analysis is realized, but this operation requires the user to introduce the parameters of binarization, noise reduction, morphological analysis and others (Fig. 7). The implementation of this approach is considered in the paper [15].

\section{Conclusion}

The paper considers the main aspects of image processing and analysis in the studying the corrosion damage mechanism:

- The computer vision and image processing in the problem of detecting bubbles on the aluminum surface are developed;

- The general regularity of corrosion data processing on a aluminum surface is defined;

- The described algorithms are used for detection and recognition of corrosion damage for an aluminum sample by observing the intensity of hydrogen bubbles formation; 
- The trained classifier was successfully applied to study several experiments of corrosion on the aluminum surface.

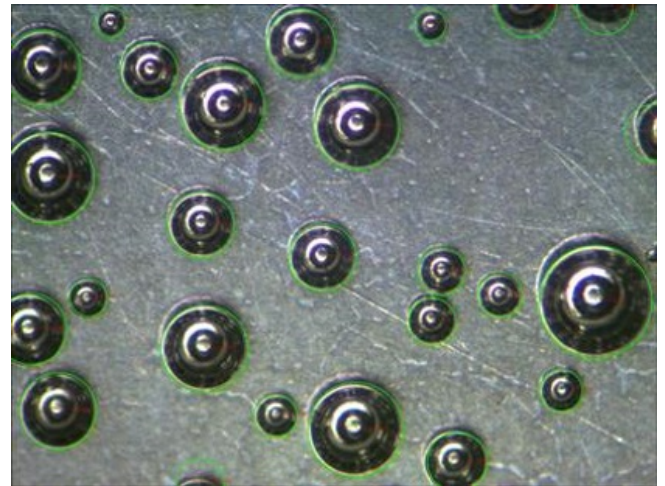

Figure 7. The work of the bubble detector on the aluminum surface.

\section{References}

[1] Weisner M, Ihlemann J, Muller H, Lankenau E and Huttmann G 2010 Optical Coherence Tomography for Process Control of Laser Micromaching Rev. Sci. Instrum. 81033705

[2] Kotenev V 2001 Optical scanning of morphology of the protective layers and forecasting of corrosion resistance thermooxidized steel Protection of Metals 37(6) 565-577 (in Russian)

[3] Weber M 2011 Confocal microscopy of the micro and nanostructured surfaces of materials Nanotech Industry 28(4) 42-47 (in Russian)

[4] Kamsu-Foguem B 2012 Knowledge-based support in Non-Destructive Testing for health monitoring of aircraft structures Advanced Engineering Informatics 26 859-869

[5] Itzhar D, Dinstein I and Zilberberg T 1981 Pitting corrosion evaluation by computer image processing Corrosion Science 21(1) 17-22

[6] Quinn M, Bailey M, Ikeda B and Shoesmith D 1993 Image-analysis techniques for investigating localized corrosion processes Atomic Energy of Canada Limited p 52

[7] Yan Y, Gao J, Liu Y and Lei S 2002 Recognition and classification of metal fracture surface models based on wavelet transform Acta Metallurgica Sinica 38(3) 309-314

[8] Rudakova O 2012 Fraktal'nyy podkhod k analizu osobennostey ustalostnogo razrusheniya svarnykh shvov Bulletin of the Perm National Research Polytechnic University. Mechanical Engineering, Materials Science 14 102-107 (in Russian)

[9] Abdel-Qader I, Abudayyeh O and Kelly M 2003 Analysis of edge-detection techniques for crack identification in bridges Journal of Computing in Civil Engineering 17(4) 255-263

[10] Yamaguchi T, Nakamura S, Saegusa R and Hashimoto S 2008 Image-Based Crack Detection for Real Concrete Surfaces IEEJ Trans Elec Electron Eng 3 128-135

[11] Prasanna P, Dana K, Gucunski N and Basily B 2012 Computer-vision based crack detection and analysis Proceedings of SPIE - The International Society for Optical Engineering 8345115

[12] Abdel-Qader I, Pashaie-Rad S, Abudayyeh O and Yehia S 2006 PCA-Based algorithm for unsupervised bridge crack detection Advances in Engineering Software 37(12) 771- 778

[13] Lattanzi D and Miller G 2014 Robust automated concrete damage detection algorithms for field applications Journal of Computing in Civil Engineering 28(2) 253-262

[14] Kantola K and Tenno R 2009 Machine vision in detection of corrosion products on SO2 exposed ENIG surface and an in situ analysis of the corrosion factors Journal of Materials Processing Technology 209(5) 2707-2714

[15] Belim S and Kutlunin P 2015 Boundary extraction in images using clustering algorithm Computer Optics 39(1) 119-124 DOI: 10.18287/0134-2452-2015-39-1-119-124

[16] Abulhanov S, Popov S, Ivliev N and Podlipnov V 2017 Device for Control of Apertures Surface of Pipes of Oil Assortment Procedia Engineering 176 645-652

[17] Vasin N and Diyazitdinov R 2016 A machine vision system for inspection of railway track Computer Optics 40(3) 410-415 DOI: 10.18287/2412-6179-2016-40-3-410-415

[18] Enikeev M, Gubaydullin I and Maleeva M 2017 Analysis of corrosion process development on 
metals by means of computer vision Engineering Journal 21(4) 183-192

[19] Dalal N and Triggs B 2005 Histograms of Oriented Gradients for Human Detection Proceedings of the IEEE Computer Society Conference on Computer Vision and Pattern Recognition 1 886-893

[20] Vapnik V 1999 An Overview of Statistical Learning Theory IEEE Transactions on Neural Networks 10(5) 988-999 